\title{
CONTRATACIÓN LOGÍSTICA EN COLOMBIA: IMPLEMENTACIÓN DE UN OPERADOR LOGÍSTICO INTEGRAL
}

\author{
Recibido: 22 de enero de 2015 - Aprobado: 10 de noviembre de 2015
}

John Alexander González Correa**

\section{RESUMEN}

Este artículo tiene como objetivo presentar los criterios que se deben tener en cuenta para contratar los servicios de los operadores logísticos. Las evidencias presentadas en este artículo se basan en información obtenida a través de encuestas, entrevistas, estudio de casos, sondeos y revisión de literatura. La principal conclusión muestra que la claridad contractual es clave en el surgimiento de un nuevo modelo de negocio que puede impulsar el desarrollo del país. Las empresas deben ver la contratación logistica con una oportunidad estratégica, al igual que pensar en cada posible situación que se pueda presentar para así plasmar un curso de acción en el contrato.

\section{PALABRAS CLAVE}

Logística del transporte, operadores logísticos, contratación intermodal, contratación multimodal, logística integral, tercerización.

\section{CLASIFICACIÓN JEL}

R41, R49, L91.

\section{CONTENIDO}

Introducción; 1. Evolución de la contratación logística; 2. Implementación de un operador logístico; 3. Recomendaciones en la implementación de un operador logístico; 4. Conclusiones; Bibliografía.

* Este artículo de reflexión es producto del proyecto "Simplificación, optimización y gestión de las relaciones y procesos contractuales de los servicios asociados al comercio exterior en la unidad de compras: Implementación de un operador logístico integral", formulado en la práctica empresarial en las Empresas Pública de Medellín E.S.P., durante primer semestre de 2013.

** Profesional en Negocios Internacionales, Universidad de Medellín, Medellín, Colombia. Consultor Hunter, Avantel S.A.S. Dirección: Carrera 50 D \# 77 Sur 81, La Estrella-Antioquia. Tel: (57) (4) 3381672. Correo electrónico: Jhohn.Gonzales.Correa@gmail.com 


\section{LOGISTICS OPERATION HIRING PROCESS IN COLOMBIA: THE IMPLEMENTATION OF A CROSSCUTTING LOGISTICS OPERATOR.}

\section{ABSTRACT}

The main objective of this paper is to present opinions that must be bared in mind when hiring services from logistics operators. The evidence presented in this paper was obtained from information gathered from surveys, interviews, case studies, polls, and literature reviews. The main conclusion shows that contractual clarity is key during the upsurge of a new business model that can drive the development of the country. Businesses must see the hiring of logistics services as a strategic opportunity as well as listing all the possible situations that can arise in order to include in the contract a course of action to be followed.

\section{KEY WORDS}

Transportation logistics, Logistics operators, Intermodal hiring, , Crosscutting logistics, outsourcing

\section{JEL CLASSIFICATION}

R41, R49, L91.

\section{CONTENT}

Introduction; 1. Evolution of logistics services hiring; 2. Logistics operator implementation; 3. Recommendations when implementing a logistics operator; 4. Conclusions; Bibliography.

\section{CONTRATAÇÃO LOGÍSTICA NA COLÔMBIA: IMPLEMENTAÇÃO DE UM OPERADOR LOGÍSTICO INTEGRAL}

\section{RESUMO}

Este artigo tem como objetivo apresentar os critérios que se devem ter em conta para contratar os serviços dos operadores logísticos. As evidências apresentadas neste artigo se baseiam em informação obtida através de enquetes, entrevistas, estudo de casos, sondagem e revisão de literatura. A principal conclusão mostra que a claridade contratual é chave no surgimento de um novo modelo de negócio que pode impulsar o desenvolvimento do país. As empresas devem ver a contratação logística com uma oportunidade estratégica, ao igual que pensar em cada possível situação que se possa apresentar para assim plasmar um curso de ação no contrato.

\section{PALAVRAS CHAVE}

Logística do transporte, operadores logísticos, contratação intermodal, contratação multimodal, logística integral, terceirização.

\section{CLASSIFICAÇÃO JEL}

R41, R49, L91.

\section{CONTEÚDO}

Introdução; 1. Evolução da contratação logística; 2. Implementação de um operador logístico; 3. Recomendações na implementação de um operador logístico; 4. Conclusões; Bibliografia. 


\section{INTRODUCCIÓN}

Según Tompkins Associates (2010), un operador logísticol es una empresa dedicada a prestar servicios integrales de logística en la cadena de abastecimiento. Muchos de ellos se encargan de la totalidad de la logística de las empresas con las que contratan, mientras otros ofrecen sus servicios por unidades de negocio.

Los operadores logísticos son una modalidad de contratación reciente en el país que viene posicionándose en el mercado gracias a la eficiencia de las operaciones logísticas, las economías de escala, la simplificación de las operaciones en la cadena de abastecimiento, el cambio de costos fijos por variables y la liberación de capital interno con el fin de dedicarlo al negocio principal de la empresa contratante.

En la década de 1980, este tipo de empresas ni siquiera existían en el entorno nacional; sin embargo, la globalización y la competencia en el mercado local, que se ha traducido en el arribo de multinacionales a Colombia, ha forzado a evolucionar los procesos logísticos a tal forma que, hoy en día, según Vargas (2013), la contratación en la logística alcanza el $40 \%$ al interior de país. Quizás, en otras dos décadas, la tendencia sea la regla general.

Acorde a la creciente relevancia de los operadores logísticos en Colombia, el presente artículo ilustra sobre la viabilidad de contratar a los operadores logísticos, el alcance que deben tener y las condiciones contractuales a pactar. El artículo se basa en las principales conclusiones de un estudio donde se aproxima la temática desde el ámbito legal, administrativo, de mercado y financiero con el objetivo de analizar la viabilidad de implementar un operador logístico en la empresa y el impacto que esto conllevaría internamente.

Aunque en general se demostró que un operador logístico crea sinergias dentro de la cadena de suministro de la empresa, esta no es una figura que se adapte por completo a todas las empresas, su implementación debe seguir un proceso riguroso de análisis sobre el modelo de negocio de la compañía y sus necesidades específicas en la cadena de abastecimiento.

En este texto podrá encontrar un análisis de los aspectos más relevantes referentes a la contratación de un operador logístico. El artículo está divido en cuatro partes. La primera parte, evolución de la contratación logística, explica la génesis de la contratación logística y las dinámicas que han marcado su evolución. En el segundo apartado, implementación de un operador logístico, se detalla la figura del operador logístico en Colombia y se establecen criterios para su escogencia y alcance de delegación. En la tercera sección, se presentan las recomendaciones finales para

1 También conocido o asociado al nombre en Inglés de Third Party Logistics (3PL) 
implementar, contratar y evaluar al operador logístico o 3PL por sus siglas en inglés. Por último, en la cuarta sección, se muestran las principales conclusiones.

\section{EVOLUCIÓN DE LA CONTRATACIÓN LOGÍSTICA}

\subsection{Una tendencia global: concentración y delegación de los servicios logísticos}

La dinámica de la logística global y la realidad de la localidad han convergido en una glocalización ${ }^{2}$ logística, es decir, la concatenación de la siempre estandarizada logística internacional con las distintivas necesidades locales. En este panorama, las empresas tienen que estar conscientes y atentas a los cambios en toda la estructura logística global debido a que un pequeño cambio normativo, empresarial o logístico en una localidad del mundo puede tener efectos en toda la cadena de abastecimiento.

El mantenimiento de departamentos de logística internos, como unidades de apoyo al core business, implica un desgaste para las empresas debido a que deben estar atentas a los cambios en toda la estructura logística global y administrar sus activos logísticos. Estas empresas suelen tener la necesidad de emplear personal especializado en logística, lo cual va en contravía al sentido misional, más aun cuando existe la necesidad de contratar personal en el negocio principal.

El nacimiento de corporaciones globales dedicadas a proveer servicios de logística integral para otras empresas, ha ayudado a reducir presión sobre los departamentos de logística de las empresas que contratan sus servicios. Esta contratación logística es una tendencia asociada a la macro-tendencia del outsourcing en el ámbito mundial.

El outsourcing debe ser entendido como un proceso natural en el cual una compañía se desprenda de todos los procesos que no agregan valor a su cadena productiva. Es una tendencia mundial con la cual el MCIT (2013, p. 9) planea, con apoyo del BID (Banco Interamericano de Desarrollo), convertir a Colombia en un país del outsourcing.

Por otro lado, en la Encuesta Nacional de Logística-2008, Rey (2008, p.29) reconoce el papel de la tercerización en las economías de América Latina:

Las empresas que tercerizan activamente son capaces de gerenciar desempeño y no necesariamente gerenciar actividades. Estas empresas además han identificado sus competencias medulares en ciertos procesos empresariales y han delegado la ejecución de otros procesos en manos de proveedores especializados. En América Latina los países con altos índices de tercerización también han desarrollado una

2 Termino mediante el cual se explica la convergencia entre globalización y localidad, la cual sugiere que se debe adaptar lo global a lo local. Para más información véase el texto de Robertson (1995) sobre glocalización. 
Contratación logística en Colombia: implementación de un operador logístico integral

economía de servicios con mayor valor agregado y con capacidad de bajar costos vía

la absorción de volúmenes de sus clientes creando economías de escala.

Es así como la logística es vista hoy en día como uno de los últimos reductos por conquistar de la tendencia del outsourcing, entendiéndose los grandes y onerosos departamentos de seguridad y vigilancia como primeros y la producción per se cómo segunda en ser contratada.

Las grandes compañías y organizaciones siguen la tendencia. En la Formula 1, por ejemplo, según CdS (2012) los servicios de logística son prestados por un solo operador. La compañía DHL se encarga del transporte terrestre, aéreo y acuático de todos los suministros mecánicos y personal humano de todas las escuderías, desde Ferrari hasta McLaren.

\subsection{Proceso colombiano}

En Colombia este proceso ha representado grandes fusiones, adquisiciones y alianzas estratégicas entre empresas que brindaban pocos servicios de logística, para agrupar un portafolio de servicios más amplio que ofreciera soluciones de logística integral en toda la cadena de abastecimiento de sus clientes. La estrategia de cooperación entre las empresas nacionales tomó lugar como un esquema de protección y competencia frente a las empresas multinacionales que llegaron después de la apertura económica.

En el entorno nacional, se evidenciaron fusiones de corporaciones que agrupaban hasta siete empresas logísticas con diferentes razones sociales. También se puede observar en el conglomerado empresarial agrupaciones empresariales de organizaciones que prestan servicios conexos. Las alianzas estratégicas son comunes, constituidas por dos o más empresas que ofrecen servicios complementarios. En una menor proporción se puede encontrar las agencias e intermediarios, empresas que ofrecen los servicios de otras empresas. Todas estas figuras diseñadas para competir de manera más eficiente, resultaron en un beneficio para el cliente: la reducción de interlocutores en las operaciones logísticas.

En este sentido, líderes empresariales entrevistados en Semana (2013, p. 82) reconocen las ventajas de la concentración de las actividades logísticas:

La última tendencia en logística evidencia que el cliente quiere integrar sus servicios en la cadena logística y garantizar la trazabilidad de su mercancía, desde el origen hasta su destino. (...) lo que proponemos a nuestros clientes es encargarnos de toda la cadena logística a través de un solo operador y con menores costos. (...) Además [el clientel ya no tendrá que buscar cada servicio de la cadena por separado: nosotros nos encargamos de todo y eliminamos costos y sobrecostos. 
La anterior tendencia se empezó a evidenciar desde que se empezaron a ratificar tratados de libre comercio (TLC) como un esfuerzo de insertar la economía Colombiana en las dinámicas de globalización, pero según Semana $(2013$, p. 16) con mayor intensidad desde el TLC con Estados Unidos.

Este reto comercial [TLC con USA], que entró en vigencia en mayo del 2012, cambió el negocio del manejo y transporte de mercancías y las empresas de este segmento [Logística] rápidamente empezaron a prestar servicios integrales que respondieran a las necesidades de los clientes. Pasaron de llevar y entregar paquetes a consolidarse como operadores logísticos. ${ }^{3}$

En general, en los servicios de logística, se pueden observar dos tendencias: 1) los proveedores de servicios de logística (PSL) se integran verticalmente para ofrecer servicios de logística integral y 2) los generadores de carga se integran de manera horizontal para ganar poder de negociación frente a los PSL.

Sin embargo, Semana (2013, p. 25), reconoce que la mayoría de empresas en Colombia aún no utilizan los servicios tercerizados de logística integral mediante operadores logísticos, los cuales son comunes en regiones más desarrolladas como en Asia, Europa y Norte América donde su uso es la regla porque los exportadores siempre quieren una sola cadena logística puerta a puerta

Es apenas normal que Colombia esté atrasado en el desarrollo logístico, puesto que este va estrechamente ligado al nivel de tercerización de la economía, el cual a su vez es proporcional al desarrollo del país. En este sentido Klein (2002) argumenta que en los países desarrollados el outsourcing está muy consolidado e impregnado en la economía. Compañías como Nike se han dedicado al core business de su empresa, es decir al diseño y mercadeo, liberándose de la producción, logística y demás funciones que pueden ser cumplidas por terceros y que no añaden valor en a la empresa.

\subsection{Etapas de la contratación logística}

La tendencia puede ser fragmentada en cuatro etapas claves: medios propios, contratación intermodal, contratación multimodal y la delegación de la cadena de abastecimiento en manos de un operador logístico integral. Cada etapa implica mayor delegación y menor responsabilidad de la empresa contratante.

La transición de una etapa a la otra no se realiza en un solo paso, lo más recomendado es implementar modelos paralelos que converjan en un sistema dual temporal que sirve como plataforma mientras se ajusta el nuevo modelo entrante.

3 El caso se refiere a la compañía TCC, la cual evolucionó de un courier a un operador logístico con la capacidad de manejar todo tipo de carga en cualquier régimen, de importación o exportación, y para una amplia variedad de destinos y orígenes. 
Algunas empresas, por ejemplo, mantienen control de las operaciones más complejas mientras delegan las menos estratégicas gradualmente.

Por otro lado, hay negocios, como las grandes superficies, en los cuales se requieren medios propios (bodegas y/o camiones) que no pueden ser delegados; sin embargo, en este caso es muy común que los activos logísticos sean administrados por operadores logísticos con personal in-house en las instalaciones de la empresa contratante ${ }^{4}$.

Otras empresas, como las de servicios públicos, operan bajo un sistema dual debido a la naturaleza de sus operaciones: un problema sanitario debe ser atendido con la mayor de las urgencias y por medios propios que la empresa pueda controlar sin importar el costo. En este último caso, esas operaciones hacen parte del core business.

A medida que se avanza en cada etapa, aumenta el nivel de delegación en la empresa contratista mientras disminuye la responsabilidad de la empresa contratante. Lo anterior implica la trasferencia de la administración y coordinación de la cadena de abastecimiento por partes hasta alcanzar el máximo en el cual la empresa contratante solo se preocupará por controlar al contratista 3PL (ver gráfica 1).

Grafica 1. Pirámide de delegación y responsabilidad

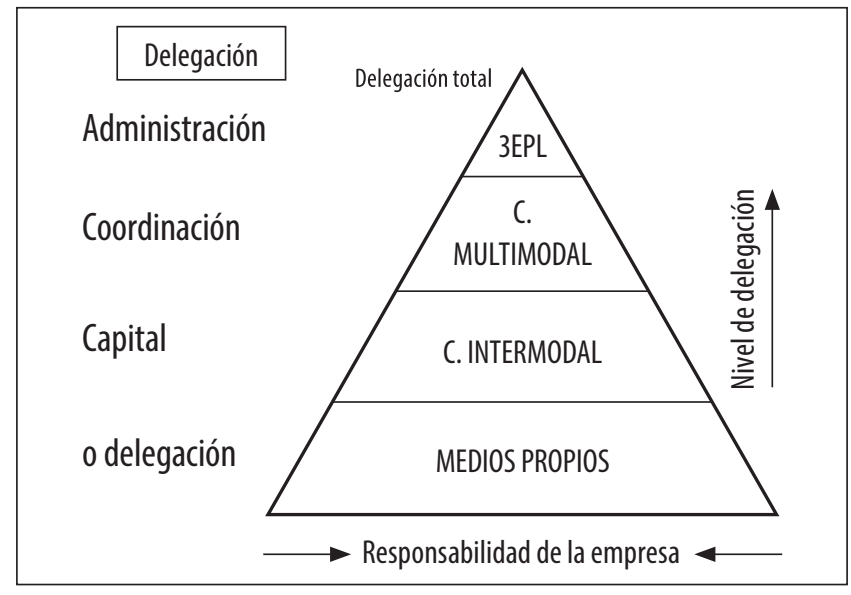

Fuente: elaboración propia

Como ya se mencionó, una empresa puede estar en más de una etapa porque está en un proceso de mutación o transitorio, es decir, se intenta pasar de una etapa a la otra, o está en varias etapas porque la naturaleza de su negocio así lo demanda como es común en las grandes superficies o en las empresas de servicios públicos 5 .

4 El centro de distribución de Almacenes Éxito en el municipio de Envigado, por ejemplo, cuenta con personal y asistencia logística de diferentes operadores logísticos.

5 Empresas Públicas de Medellín es un buen ejemplo de este modelo. 


\subsubsection{Etapa 1: medios propios}

La mayoría de compañías invertían cuantiosas sumas de capital en medios de transporte y activos logísticos para realizar sus propias operaciones, esto era justificado por la poca oferta de compañías de transporte y logística. Aunque este modelo sigue vigente hoy en día para algunas compañías, el grueso de las empresas contratan a otras para transportar sus mercancías y, algunas, para almacenarlas y llevar a cabo operaciones de logística integral.

La utilización parcial o total de activos logísticos propios es justificada en algunos casos específicos. Existen, por ejemplo, empresas que utilizan un operador logístico propio ${ }^{6}$, el cual se encarga de la totalidad de la logística de la empresa. Esto es justificado por las características únicas de sus productos (como el cemento) que demandan especificaciones técnicas muy exigentes en el transporte. Incursionar en este tipo de nichos de mercado es muy costoso para las empresas de transporte externas, sobre todo cuando pueden obtener mayores réditos con la misma inversión en mercados de carga general.

Este modelo es viable cuando las especificidades de la carga o las necesidades del negocio así lo ameriten. Combinaciones entre los modelos son posibles y funcionan. Se denota que estas son excepciones a la regla. Las compañías de transporte, en general, son más eficientes en sus procesos debido a que este es su core business mientras que en las demás empresas, el transporte es un proceso de suplementario.

\subsubsection{Etapa 2: contratación intermodal ${ }^{7}$}

La contratación intermodal es aquella en la que se contrata cada modo de transporte por separado. En esta modalidad, se procura la elaboración y perfeccionamiento de tantos contratos como modos de transporte vaya a utilizar la empresa (ver gráfica 2). Por consiguiente; si en las operaciones de transporte están involucrados al menos dos modos de transporte, se debe procurar la elaboración de al menos dos contratos.

La coordinación logística es realizada por un departamento interno de la empresa contratante. El equipo interno debe coordinar entre sí a los contratistas involucrados en cada modo, con el fin de que se pueda realizar la operación a cabalidad, esto

6 La compañía de cementos Argos es un buen ejemplo de ello.

7 Todavía no hay consenso en cuanto al uso de este término y en general a los términos que hacen referencia al uso de las modalidades de transporte. Se puede apreciar que el término intermodal es fácilmente confundido con multimodalismo. La confusión radica esencialmente en la diferencia entre medio y modo de transporte. Sin embargo, por este título no se hace referencia al transporte intermodal, el cual es la utilización de varios medios dentro del mismo modo, sino a la manera de contratar la logística. Para una aclaración de la palabra intermodal remítase al artículo de Ruiz (2013). 
incluye el envío de innumerable cantidad correos donde se intercambia información referente a la carga: características, trazabilidad, quien la genera, transporta, legaliza, almacena, recibe, etc.

En esta modalidad de contratación pueden intervenir, además de la empresa contratante, empresas de transporte, agentes de carga internacional (ACI), navieras, intermediarios, agencias de aduanas, proveedores de sistemas informáticos, agencias de aduanas y almacenes generales, entre otros PSL.

Grafica 2. Flujo contractual en el transporte intermodal

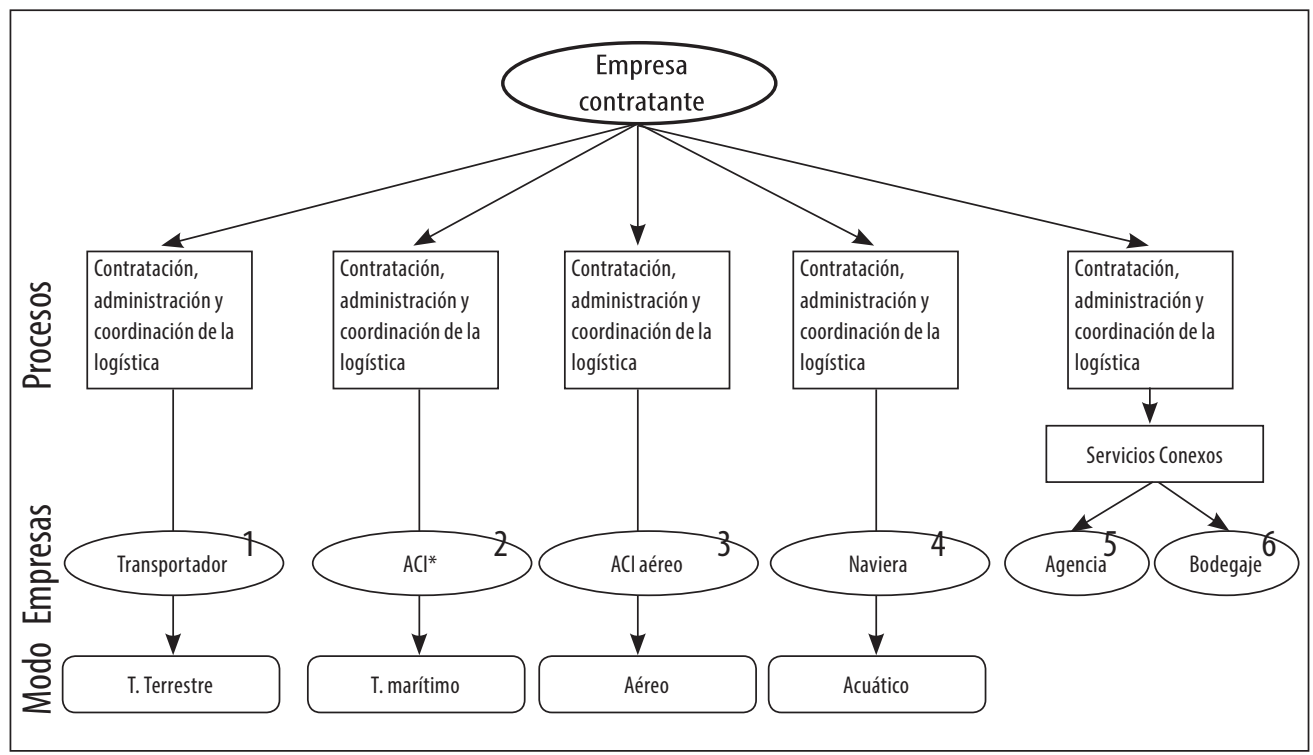

* Agentes de Carga Internacional

Fuente: elaboracion propia

Esta modalidad conlleva ciertas ventajas frente a la anterior de medios propios; sin embargo, implica más desventajas que ventajas ante las etapas que le prosiguen. A medida que se avanza más en el texto se entenderá el porqué de esta aseveración.

Algunas de las ventajas de esta etapa son:

- Liberación de capital que antes era retenido en medios propios, a favor de capitalizar esos recursos en el negocio principal de la empresa contratante.

- No se requiere tanto personal especializado en comparación con la etapa anterior. El capital de trabajo se reduce en la empresa contratante y aumenta el nivel de delegación en las empresas de logística. 
- La responsabilidad y los riesgos son distribuidos en varios contratistas que se encargan de ejecutar las actividades. Si se sabe distribuir los servicios en diferentes contratistas se puede disminuir los riesgos.

\subsubsection{Etapa 3: contratación multimodal}

La contratación multimodal es aquella por medio de la cual se concentra los di ferentes contratos de transporte en un solo contrato otorgado a un proveedor de servicios logísticos, el cual puede ser, según el mercado colombiano, un agente de carga internacional, un operador logístico, un intermediario o cualquier empresa certificada como operador de transporte multimodal (OTM).

Los OTM surgen en respuesta a la tendencia expuesta al principio del capítulo. Este operador es, grosso modo, una empresa que se encarga de transportar la carga de otra por al menos dos modos de transporte bajo un mismo contrato de transporte denominado contrato de transporte multimodal (ver gráfica 3). La revista Semana (2013, p. 22) los define como: "Una carga, un transportador, varios modos de transporte que se complementen y una empresa responsable de toda la operación hacen parte de la filosofía del multimodalismo"

Grafica 3. Flujograma contratación multimodal

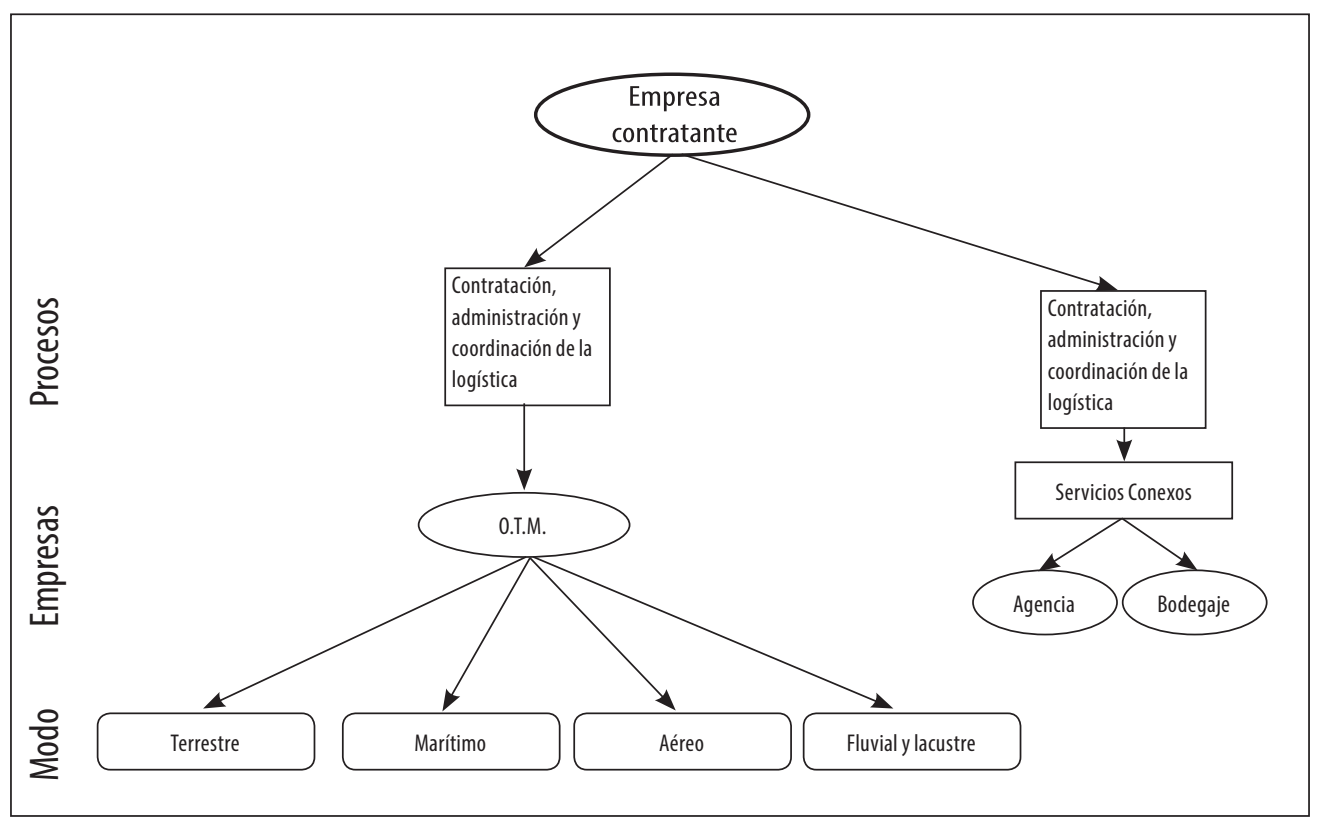

Fuente: elaboración propia 
Algunos beneficios de la contratación multimodal son:

- Aprovechamiento del mayor conocimiento del mercado, experticia en las actividades y know-how del OTM

- Simplificación de las operaciones logísticas, contractuales y administrativas de la empresa contratante, debido a la existencia de un solo contrato de transporte y un solo interlocutor para el transporte con responsabilidad total.

- Mayor poder de negociación de la empresa contratante. Los servicios de transporte antes demandados por separado se agrupan para así generar contratos más grandes y atractivos frente al conglomerado empresarial logístico.

- Mejores ofertas del proveedor de servicios logísticos. Las potenciales economías de escala permiten abaratar costos y bajar los precios de transporte.

- Mayor coordinación en la cadena de abastecimiento. La concentración de la operación en un solo PSL permitirá sincronizar procesos y actividades que antes no se podían por la falta de integración en la cadena de abastecimiento. Cuando hay varios PSL en una misma cadena, estos no comparten información entre sí en la mayoría de los casos por suponer que la información no es relevante, no hay confianza o porque no existen canales de comunicación entre ellos.

- La coordinación entre despachos, buques, barcazas y camiones permite eliminar tiempos muertos en el tránsito, llevar la trazabilidad de la carga con mayor precisión y reducir el tiempo de almacenamiento vía tiempos de tránsito más cortos. Si la sincronización alcanza proporciones importantes, se puede llegar a operaciones just in time y así reducir el inventario.

- Según el Decreto 2685 (1999) las operaciones realizadas en esta modalidad gozan de ciertos beneficios tributarios y aduaneros en Colombia. La carga se mueve con tributos suspendidos en continuación de viaje y el tiempo en puerto es menor.

Aunque en el mercado colombiano muchos operadores logísticos son OTM, no todos los OTM son operadores logísticos. Estos últimos ofrecen en sus portafolios, servicios adicionales de bodegaje, agenciamiento aduanero, informática especializada, entre otros.

\subsubsection{Epata 4: operador logístico integral (3PL)}

De acuerdo a Tompkins Associates (2010, p. 2) "Un operador logístico integral es la utilización de una compañía externa para llevar a cabo algunas o todas las funciones de la 
cadena de abastecimiento que una organización requiere" Este es el máximo nivel de tercerización logístico alcanzado en Colombia, puesto que los 4PL ${ }^{8}$ (Fourth Party Logistics) son una rareza en el país y la última frontera de la logística global.

El alcance de un 3PL incluye, entre otros servicios, transporte, aduana, almacenaje, distribución, gestión de compras ${ }^{9}$, asesoría tecnológica y administrativa.

La empresa que delega las actividades logísticas a este tipo de compañías encuentra varios beneficios que repercuten en la optimización de la cadena de abastecimiento. Algunos son:

- La coordinación de sus actividades se simplifica aún más gracias a que se relaciona con una sola compañía. La comunicación es bilateral y no multilateral como ocurre cuando existen varias compañías involucradas en el proceso (ver gráfica 4).

- La delegación de la responsabilidad, coordinación y parte de la administración de los procesos logísticos y de comercio exterior implica una disminución en la carga de trabajo que la empresa contratante puede dedicar al negocio esencial de su actividad.

- Mayor control de los costos asociados a la logística. La estructura de costos es completa y se sabe a cabalidad todos los servicios que serán prestados, al igual que sus tiempos. Del mismo modo, una sola factura y un solo contrato simplifica el extensivo papeleo que se requiere en el comercio exterior y en la contratación.

- Otros beneficios que permiten los 3PL con contratos estables y a largo plazo son los desarrollos tecnológicos y la integración de plataformas tecnológicas que un 3PL puede realizar con su cliente, para optimizar las operaciones entre las partes. Generar eficiencias en las operaciones del cliente implica ganancias monetarias para ellos y de servicio para la empresa contratante. Estos beneficios pueden incluir desarrollos de sistema de administración de bodegas integrados (WMS, por sus siglas en Inglés) e intercambio electrónico de datos (EDI, por sus siglas en Inglés) entre las diferentes plataformas tecnológicas de las empresas.

8 Externalización más amplia, el operador se responsabiliza de la optimización de una cadena global donde se incluye el cliente, los clientes de sus clientes y los proveedores de sus proveedores.

9 El Ministerio de Relaciones Exteriores de Colombia, en el año 2014, por ejemplo, implementó un 3PL para gestionar las compras de la entidad, con el fin de agilizar la contratación. 
- La estructura de costos cambia. Reemplazar costos fijos por costos variables es una consecuencia natural del outsourcing. Una organización más flexible es posible gracias al nuevo modelo, permitiéndole a la empresa ajustarse a eventos inesperados como mercados volátiles o fluctuantes.

- Los 3PL que subcontratan parte de los procesos tienen la libertad de escoger el mejor agente del mercado según las necesidades específicas del cliente, lo que asegura el mejor trato posible. Este tipo de LSP poseen un mayor poder de negociación ante terceros debido a que tienen a cargo mercancía de varios clientes. Los 3PL que subcontratan parte de las operaciones también tienen dentro de las instalaciones personal in-house de los subcontratistas, un lujo imposible de tener en una empresa con un bajo flujo de carga. Estos in-house permiten una mejor coordinación y eficiencia en las operaciones.

- Según Langley $(2013$, p. 9) los generadores de carga en el contexto mundial reconocen el mayor rendimiento asociado a la subcontratación logística: la reducción de costos logísticos (15\%), la reducción de costos de inventarios (8\%), la reducción de activos logísticos fijos (26\%), el mejoramiento del índice de procesamiento de ordenes (De 58\% a 65\%) y la exactitud de las ordenes (De $67 \%$ a $72 \%)$

\section{Grafica 4. Flujo contractual en la logistica Integral}

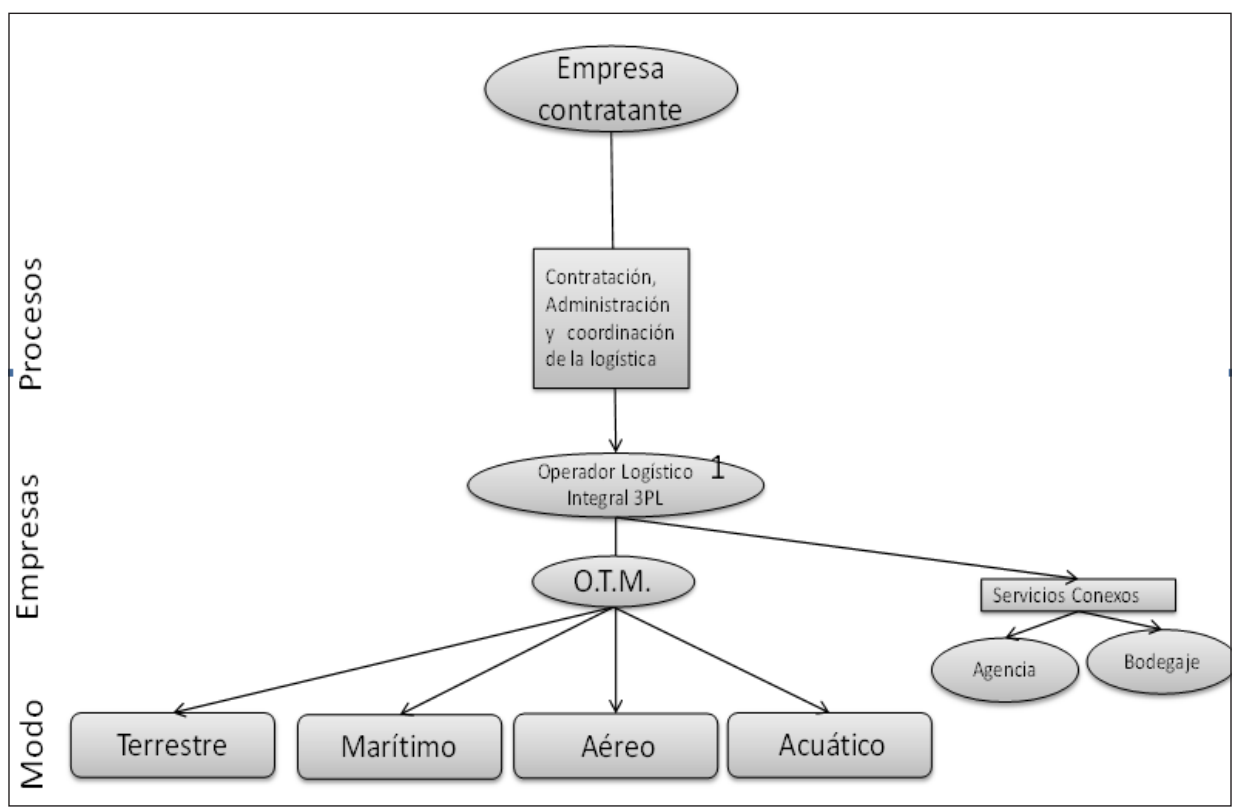

Fuente: elaboración propia 


\section{IMPLEMENTACIÓN DE UN OPERADOR LOGÍSTICO}

\subsection{Clasificación de los operadores logísticos}

Según la firma de consultoría en logística Tomkins Associates (2010, p. 14), existen tres tipos de 3PL:

1) Basados en el capital, ofrecen los servicios de logística por medio de activos propios.

2) Basados en el manejo de proveedores, no poseen los medios de transporte ni almacenaje. Subcontratan las operaciones con terceros.

3) Integrados, complementan los servicios con los de otras empresas. Son una mezcla entre los primeros y los segundos.

Es vital conocer los tipos de 3PL debido a que a la hora de seleccionar se puede caer en el error de limitar la participación de algunos agentes del mercado justificándose en la estructura y modelo de negocio. No se debe subestimar la capacidad de aquellos 3PL que no cuentan con medios propios para llevar a cabo la operación. El equipo de trabajo, el know-how, las conexiones y ventajas competitivas son la base fundamental de un 3PL, no los activos.

\subsection{Figuras legales de 3PL según su composición empresarial en Colombia}

El modelo de negocio de los operadores logísticos puede variar según la composición empresarial. Si un 3PL no presta todos los servicios, este se complementa de otras compañías: Empresas del mismo grupo empresarial (filial, subsidiaria o matriz), aliados estratégicos y/o subcontratistas (ver tabla 1).

Por ende es indispensable que un eventual contrato contemple todas estas configuraciones posibles, no solo la participación individual.

Tabla 1. Estructura empresarial de los 3PL

\begin{tabular}{|l|c|c|c|c|c|c|c|c|c|l|}
\hline \multicolumn{1}{|c|}{ Empresa } & Orden & A.C.I & Naviera & Aerolínea & Trans. terrestre & Almacén & O.T.M. & Agencia & Clasifica & \multicolumn{1}{c|}{ Participación } \\
\hline Empresa A & Nacional & $\mathrm{O}$ & & & $\mathrm{O}$ & $\mathrm{X}$ & & $\mathrm{X}$ & 1 & Grupo empresarial \\
\hline Empresa B & Nacional & $\mathrm{X}$ & & & $\mathrm{X}$ & $\mathrm{X}$ & & & 1 & Empresa unitaria \\
\hline Empresa C & Nacional & $\mathrm{X}$ & & & $\mathrm{X}$ & $\mathrm{X}$ & & $\Omega$ & 1 & Empresa unitaria \\
\hline Empresa D & Multinacional & $\mathrm{X}$ & $\Omega$ & & $\Lambda$ & $\Lambda$ & & $\Omega$ & 2 & 3pl. sin activos fijos \\
\hline Empresa E & Nacional & $\mathrm{X}$ & $\mathrm{X}$ & & $\Omega$ & $\mathrm{X}$ & & & 1 & consorcio \\
\hline Empresa F & Multinacional & $\mathrm{X}$ & $\mathrm{X}$ & $\mathrm{X}$ & $\mathrm{X}$ & $\mathrm{X}$ & $\mathrm{X}$ & $\mathrm{X}$ & 1 & Empresa unitaria \\
\hline Empresa G & Multinacional & $\mathrm{X}$ & & & $\Lambda$ & $\mathrm{X}$ & $\mathrm{X}$ & $\mathrm{X}$ & 3 & Empresa unitaria \\
\hline
\end{tabular}


Contratación logística en Colombia: implementación de un operador logístico integral

\begin{tabular}{|c|c|c|c|c|c|c|c|c|c|c|}
\hline Empresa & Orden & A.C.I & Naviera & Aerolinea & Trans. terrestre & Almacén & O.T.M. & Agencia & Clasifica & Participación \\
\hline Empresa $\mathrm{H}$ & Nacional & $X$ & & & $\mathrm{O}$ & $\mathrm{O}$ & $\mathrm{O}$ & $\mathrm{O}$ & 1 & Grupo empresarial \\
\hline Empresa I & Multinacional & $\mathrm{X}$ & & & $\Lambda$ & & $\mathrm{X}$ & $X$ & 3 & Empresa unitaria \\
\hline Empresa J & Nacional & $\Omega$ & & & $\Omega$ & $X$ & & $X$ & 3 & Unión temporal \\
\hline Empresa K & Nacional & $X$ & $x$ & $X$ & $X$ & $X$ & $X$ & $X$ & 1 & Empresa unitaria \\
\hline Empresa L & Nacional & & & & $\mathrm{X}$ & $\Omega$ & $X$ & $\mathrm{X}$ & 1 & Alianza estratégica \\
\hline \multicolumn{11}{|c|}{ CONVENCIONES } \\
\hline $\mathrm{X}$ & \multicolumn{10}{|c|}{ Servicios propioas: las operaciones son realizadas directamente por la empresa contratada } \\
\hline 0 & \multicolumn{10}{|c|}{ Servicios prestados por filiales: la empresa matriz tienen filiales que prestan servicios conexos } \\
\hline$\Omega$ & \multicolumn{10}{|c|}{ Servicios prestados por aliados estratéticos: este tipo de alianzas puede convertirse en uniones temporales } \\
\hline$\Lambda$ & \multicolumn{10}{|c|}{ Servicios subcontratados } \\
\hline 1 & \multicolumn{10}{|c|}{ 3PL basados en el capital } \\
\hline 2 & \multicolumn{10}{|c|}{ ePL basados en manejo de proveedores: utilizan otras empresa para realizar las operaciones logísticas } \\
\hline 3 & \multicolumn{10}{|c|}{ 3PL integrados: incluye combinación de capital y manejo de proveedores logísticos } \\
\hline
\end{tabular}

Nota aclaratoria: los nombres de las empresas se cambian por nombres ficticios.

Fuente: elaboración propia.

Algunas de estas empresas 3PL incluso pueden llegar a ser Comercializadoras Internacionales $(\mathrm{CI})^{10}$. Esta característica les permite encargarse de la gestión de compras de sus clientes en el ámbito global. Existen operadores que se encargan de gestionar las compras de las PYME con lo cual el operador logra consolidar un poder de negociación ante los proveedores de sus clientes gracias a la agrupación de las compras de varios de ellos.

Hay que tener en cuenta que la calificación de una empresa como 3PL es un proceso subjetivo que depende de la apreciación del evaluador y de las mismas compañías ya que la figura no está regulada en la legislación colombiana como si lo están las figuras de ACI, O.T.M, A.D.A (Almacenes de Depósito Aduanero) y las agencias de aduana. Según Tompkins Associates (2010, p. 15) "Se debe tener precaución porque cada vez más compañías se están autonombrando como operadores logísticos, varían desde las pequeñas empresas familiares de almacenamiento hasta sofisticados conglomerados empresariales".

\subsection{Profundidad y alcance de las operaciones de logística integral}

Un operador logístico integral tiene un alcance más amplio de las operaciones de transporte, agenciamiento aduanero y bodegaje. Ellos también pueden encargarse del almacenaje o parte de este, de los sistemas de compras, la distribución de las

10 Véase Semana (2013), donde se evidencia un ejemplo de este caso. 
mercancías, implementación de sistemas logísticos, adquisición y asesoramiento, entre otras operaciones de la empresa contratante.

Por consiguiente, la profundidad de la relación con dichas empresas debe estar limitada por la capacidad de la empresa a realizar ciertos procesos logísticos, al igual que la importancia de algunos de los procesos que pueden ser considerados estratégicos o muy complicados para delegar, por ejemplo, la contratación de las compras en el sector público.

Independiente del alcance, es aconsejable realizar la delegación por fases, delegar de las operaciones más externas y comunes a las más internas y complicadas o según Langley (2013, p. 10) de las operaciones tácticas a las estratégicas, de las más a las menos tercerizadas. Esto con el fin de que si hay algún percance, se pueda corregir o la empresa contratante pueda cambiar de 3PL en una fase de las operaciones donde no haya tanta dependencia. La estrategia consiste de un involucramiento lento y progresivo donde las partes puedan conocer sus verdaderas necesidades, limitaciones, debilidades y fortalezas.

La primera fase se constituye de relaciones más externas en la cadena de abastecimiento, es decir, el transporte, el agenciamiento aduanero y gastos conexos a estas operaciones. La segunda se compone de la distribución y almacenamiento, sea este último interno o externo, lo que puede implicar la delegación de la administración de las bodegas del cliente o el almacenamiento en bodegas del 3PL o contratista de este último. La última fase está compuesta de la gestión de compras de la compañía, realizar las compras y abastecer la compañía con los suministros necesarios según los requerimientos hechos. Adicional se puede pensar en una última fase que implemente otro tipo de servicios requeridos como financieros, conocimiento, asesoría, atención al cliente, entre otros.

Este desglosamiento por fases también está sustentado en las características del mercado. Hay más proveedores de servicios logísticos que prestan servicios de logística física (transporte, distribución, agente aduanero, almacenamiento) que aquellos que prestan servicios a la administración de la cadena de abastecimiento (compras, gestión de proveedores, planeación de producción). Según Rey (2008, p. 37) "claramente los PSL prefieren mantenerse alejados de los temas de mayor coordinación en la cadena de suministro como son los temas de compras (relaciones con proveedores), planeación de producción y operaciones comerciales". Esto también se evidencia en el nivel de tercerización. Estudios de Rey (2008) y Langley (2013) señalan que los servicios de logística física son los más tercerizados mientras que otros servicios en la cadena de abastecimiento como gestión de compras, financieros y el conocimiento aún juegan un papel secundario. 
Lo usual en una empresa es que las actividades de cada fase están a cargo de equipos diferentes dentro de la empresa, por ejemplo, la primera fase puede estar a cargo de un equipo, el almacenamiento depender de otro departamento y las compras de otro equipo. La implementación por fases brinda la oportunidad de intervenir grupo a grupo y no todos a la vez. De este modo, se puede realizar una implementación más fácil de llevar a cabo para la compañía y para el líder del proyecto. Es más fácil liderar con un equipo en desacuerdo que con todos aquellos involucrados en la cadena de suministro dentro de la empresa. Según Raja (2000, p. 237) "los departamentos internos que actualmente son responsables de estas funciones tienen que ser convencidos de la idea del outsourcing" con el fin de evitar paros en el proceso.

\subsection{Escogencia de un operador logístico}

Tener contratos con compañías líderes en el mercado es sinónimo de respaldo, solidez y confianza en todas las operaciones. Confiar la cadena de suministro a una compañía externa no es una tarea fácil y debe ser delegada solo a los mejores proveedores. Cualquier inexperiencia repercute en la cadena de abastecimiento y esto puede significar interrumpir las operaciones de la empresa por desabastecimiento. La operación en cuestión debe ser delegada solo a los líderes del mercado.

Aunque sea aconsejable contratar a los líderes del mercado, esto no se debe tomar como un axioma. Es aconsejable perfilar las necesidades concretas de la empresa y entrar en un proceso exhaustivo de revisión de las fortalezas y debilidades de los agentes del mercado que puedan satisfacer esas necesidades.

Contratar a un operador logístico líder en el mercado es, en la mayoría de los casos, una relación de sometimiento a un gigante de la industria, a no ser que la contraparte sea un gran generador de carga que logre nivelar o invertir la relación de poder.

Por otro lado, contratar con un operador logístico pequeño puede conllevar una relación de apareamiento donde las partes se pueden sentar a negociar. Las organizaciones más pequeñas tienden a ser más flexibles, lo cual es muy importante cuando se manejan operaciones de comercio exterior que son onerosas, rígidas, sobre documentadas y complejas.

Otro factor clave a la hora de escoger es la configuración de los servicios ofrecidos. Al respecto Raja (2000, p. 240) argumenta que "no todos los operadores logísticos son expertos en todas las funciones logísticas". Los 3PL suelen ser fuertes en los servicios que requiere el segmento de mercado en el que se enfocan. Existen por ejemplo operadores logísticos que apuntan a nichos especializados como el transporte 
sobredimensionado. Identificar las necesidades y el operador apropiado acorde a estas puede ahorrarle mucho tiempo.

Una vez se tenga conocimiento de los potenciales proveedores, es imperativo realizar procesos de selección entre las empresas candidatas para poder determinar cuál es la opción más adecuada. En este sentido, Vargas (2013) aconseja: "conocer mejor al operador logístico, es recomendable visitar las instalaciones, conocer los clientes que maneja, su capacidad y sus indicadores, como grado de exactitud de los inventarios e índice de cumplimiento".

Las certificaciones pueden ser otra variable a evaluar al momento de escoger. Esto es esencial para aquellas empresas que tienen normas de contratación muy estrictas que exigen a los proveedores ciertos estándares. En Colombia las certificaciones más comunes en el sector logístico son: ISO 9001, OHSAS 18001 y BASC. La última certificación es importante en el sector, sobre todo, porque acredita la seguridad de las operaciones en el comercio internacional en un país donde el tráfico de estupefacientes es un problema mayor.

\subsection{Criterio de glocalidad para empresas con cadenas de abastecimiento global}

Si bien saber quiénes son los líderes nacionales es un ejercicio útil, es también indispensable saber la composición internacional. Las conexiones globales de compañías multinacionales son de gran utilidad por la amplia cobertura, lo que es fundamental cuando se cuenta con una cadena de abastecimiento global y se es una compañía multilatina.

Compañías líderes en el mercado nacional e internacional son, en teoría, las más apropiadas para administrar y coordinar una cadena de abastecimiento global. Por su éxito nacional e internacional estas compañías cumplen el criterio de glocalidad. Son empresas consientes de las necesidades y complejidades locales y con conocimiento de las amplias especificaciones y requerimientos globales.

Otra opción viable para las empresas con cadenas de abastecimiento global, son los 3PL nacionales que tienen alianzas estratégicas, convenios o acuerdos comerciales con otras empresas internacionales. El agenciamiento es la configuración más común para las empresas nacionales que quieren tener conexiones e influencias globales. Aunque esta alternativa es viable, no es tan atractiva cuando se compara con la primera opción, es decir, una sola compañía con alcance global líder en el mercado nacional e internacional. 


\section{RECOMENDACIONES EN LA IMPLEMENTACIÓN DEL 3PL}

Se debe tener un solo 3PL con cobertura global con el fin de aprovechar las economías de escala y la simplificación del proceso con una sola compañía. De lo contrario se caería en la trampa de tener muchos proveedores del mismo servicio.

La contratación de dicho proveedor debe realizarse por volúmenes considerables que atraigan la atención, y con contratos estables y duraderos en el tiempo para aprovechar los beneficios de un 3PL, es decir, la eficiencia en costos y las sinergias en la cadena de abastecimiento como innovaciones, soluciones a la medida, órdenes perfectas y menores tiempos.

Los costos y la facturación de los servicios siempre constituyen un punto álgido en la relación. Para liderar con esto, existen varias alternativas:

La primera es establecer contratos abiertos y sin tarifas especificadas. Esta solución facilitaría el proceso de contratación en compañías con normas de contratación flexibles. Sin embargo, la carencia de tarifas acrecentaría el riesgo de caer en ambigüedades, desconfianza y conflictos entre las partes. Esta opción es viable cuando la empresa confía en su proveedor de servicios logísticos o cuando el entorno macroeconómico del país es inestable, es decir, existe alta fluctuación de precios y las tarifas deben ser flexibles para que las partes se puedan adaptar a las cambiantes condiciones macroeconómicas ${ }^{11}$.

Otra opción es establecer tarifas definidas que cubran ciertos orígenes y/o destinos establecidos. Se recomienda los más utilizados por la empresa. Esta estrategia es útil para las empresas con normas de contratación más estrictas o en los procesos de contratación por licitación donde los proponentes son evaluados bajo el criterio del menor valor económico. Aunque esta opción brinda tarifas claras para ciertos puntos, deja a merced de una nueva negociación las tarifas no especificadas.

También es posible incluir cláusulas en los contratos que establezcan métodos para fijar topes en las tarifas. Estos pueden ser con referencia en precios del mercado, de la empresa, los de referencia del proveedor o una combinación de ellos. La implementación de dichas estipulaciones puede ser amplia y depende de la capacidad de innovación y concertación de los negociadores para crear propuestas de valor.

Se debe tener en cuenta que la utilización de estas estrategias contractuales depende de la capacidad de la empresa para establecerlas, es decir, el poder de negociación ante la contraparte y de las disposiciones legales, sobre todo, para empresas del sector público.

11 Esto aplica en estos momentos en países como Venezuela con altas tasas de inflación. 


\subsection{Consideraciones a la relación contractual}

- Los contratos deben de ser a largo plazo debido a que toma al menos un año para que las relaciones se establezcan con firmeza y la empresa se adapte al nuevo modelo de logística integral. Mediciones deben ser llevadas desde el inicio del contrato, pero solo deben ser comparadas con el modelo anterior aquellas que se realicen después del primer año, tiempo para el cual el volátil proceso de adaptación debió de haber pasado.

- Según Tompkins Associates (2010), se debe considerar una relación que recompense al 3PL por el rendimiento, no una estructura base de costos que no sea motivadora. Debe ser una estructura combinada, es decir, por unidad, por costo fijo y un incentivo adicional por cumplir metas o mejorar el rendimiento.

- Aunque la relación sea a largo plazo, deben de haber un nuevo acuerdo cada cierto tiempo sobre lo ya pactado y acordar nuevos requerimientos de la empresa, si existen. Lo anterior debido a que las necesidades de la empresa son cambiantes. Aunque hayan negociaciones cada año no significa que no haya contacto el resto de tiempo, al contrario, las relaciones entre las partes deben de consolidarse cada vez más vía reuniones permanentes para discutir el rendimiento y otros temas como la coordinación. Es una relación que necesita constante supervisión y control

- En una empresa donde los exigentes requerimientos contractuales hacen de la contratación un procedimiento complejo y dispendioso, no se recomienda tener demasiados contratos. Bajo este esquema de rigidez contractual, se recomienda implementar un operador logístico con un contrato único y cobertura global. La implementación de un solo operador que simplifique y flexibilice las operaciones es una decisión acertada en términos administrativos para este tipo de empresas.

\subsection{Indicadores de gestión}

Determinar la creación de indicadores para la evaluación y gestión del proyecto de implementación de un 3PL es fundamental.

La medición de cualquier proyecto sirve para determinar el impacto del mismo en el entorno y evaluar su viabilidad. Los indicadores son mecanismos que proporcionan un diagnóstico de los resultados e impactos del proyecto per se. Para que los indicadores sean efectivos en medir el impacto, estos deben ser establecidos a priori. Se debe tener claridad de la situación antes y después de implementar el proyecto. 
La tabla 3, tomada del artículo de Saldarriaga (2009 p.22), tiene en cuenta los indicadores más relevantes para un equipo que pretenda implementar un operador logístico. Estos son las métricas que deberían ser establecidas para evaluar el desempeño tanto de los proveedores como del mismo equipo encargado del proceso. Algunos de estos indicadores pueden ser usados en la parte contractual para premiar o castigar al proveedor según el desempeño.

Tabla 2. Indicadores Logísticos

\begin{tabular}{|c|c|c|c|c|c|c|}
\hline $\mathrm{N}^{\circ}$ & Indicador & Descripción & Incidencia & Urgencia & Suma & Orden \\
\hline 1 & $\begin{array}{l}\text { Tiempo de trans- } \\
\text { porte. }\end{array}$ & $\begin{array}{l}\text { Número de días transcu- } \\
\text { rridos }\end{array}$ & & & & \\
\hline 2 & Entregas a tiempo. & $\begin{array}{l}\text { Desviación porcentual en } \\
\text { días contado a partir del } \\
\text { supuesto día de entrega }\end{array}$ & & & & \\
\hline 3 & $\begin{array}{l}\text { Horas extras x pe- } \\
\text { dido. }\end{array}$ & $\begin{array}{l}\text { Promedio de horas extras } \\
\text { por pedido }\end{array}$ & & & & \\
\hline 4 & $\begin{array}{l}\text { Costos del transpor- } \\
\text { te x tonelada. }\end{array}$ & $\begin{array}{l}\text { Promedio Coste del trans- } \\
\text { porte por tonelada movi- } \\
\text { lizada }\end{array}$ & & & & \\
\hline 5 & $\begin{array}{l}\text { Extra costos de la } \\
\text { operación por pe- } \\
\text { dido. }\end{array}$ & $\begin{array}{l}\text { Media de los sobrecostos } \\
\text { por pedido. }\end{array}$ & & & & \\
\hline 6 & Ordenes perfectas. & $\begin{array}{l}\text { Numero de ordenes en las } \\
\text { que la facturación fue co- } \\
\text { rrecta, entregas completas, } \\
\text { entregas a tiempo y entre- } \\
\text { gas en buenas condiciones } \\
\text { de calidad }\end{array}$ & & & & \\
\hline 7 & $\begin{array}{l}\text { Tiempo ahorrado } \\
\text { desde la implemen- } \\
\text { tación. }\end{array}$ & $\begin{array}{l}\sum \text { Del \# de horas total con- } \\
\text { sumido en el modelo ante- } \\
\text { rior menos la } \sum \text { de horas } \\
\text { consumidas en el nuevo } \\
\text { proceso. }\end{array}$ & & & & \\
\hline
\end{tabular}

NOTA:

\begin{tabular}{|c|c|}
\hline Nivel de incidencia & Nivel de urgencia \\
\hline $\begin{array}{l}\text { Grado de importancia para la consecución de los obje- } \\
\text { tivos de las empresas: } 10 \text {, aspectos fundamentales para } \\
\text { la empresa. 1, aspectos poco importantes }\end{array}$ & $\begin{array}{l}\text { Grado de urgencia de que mejore: 10, urgencia máxima; } \\
\text { 1, urgencia mínima. }\end{array}$ \\
\hline
\end{tabular}

Fuente: Tomado de Saldarriaga (2009, p. 22)

La idea de la tabla anterior es priorizar indicadores mediante un proceso grupal en un focus group con los encargados del proceso en la compañía. Estas priorizaciones 
tienen que estar acordes a las necesidades más apremiantes de la compañía de acuerdo a la naturaleza del negocio o de las operaciones. Las compañias tienden a dar más relevancia a unas variables que a otras basadas en sus requerimientos.

\section{CONCLUSIONES}

El operador logistico es una alternativa que esta acorde a las tendencias mundiales, más no es un modelo unico, su implementación debe conlllevar un analisis minucioso de las necesidades de la empresa que decida implementar sus servicios.

Esta figura es de vital importancia en aquellas compañias donde el costo humano de realizar las operaciones logisticas es alto en comparación con los costos operativos y el valor que los primeros agregan en la cadena de valor de la compañía. Los 3PL tambien son utilies cuando no se cuenta con la infraestructura logística o esta es inadecuada y/o ineficiente. Otro factor en la ecuación es la filosofia de la empresa y el modelo de negocio, es decir, si se es una compañía que se centra en la actividad principal y se libera de las actividades secundarias o de apoyo.

En la implementación es de vital importancia la participación de los funcionarios que esten al cargo del proceso a ser delegado. Ellos son los más apropiados para ayudar a perfeccionar las relaciones con un 3PL debido al conocimiento de los procesos y de lo que se necesita hacer, sin embargo, como afirma Tomkins Associates (2010) ellos deben de ser los primeros en ser convencidos del proceso de logistica integral ya que ellos son los más propicios a sabotearlo.

Debido a que los operadores logisticos se pueden involucrar por completo en la cadena de abastecimiento de una organización y se puede delegar tanta responsabilidad en ellos, es de vital importancia que el contrato que rija dicha relación entre las partes quede tan claro y completo como sea posible. El negociador debe pensar en esta relación con una visión tactica y hasta estratégica, al igual que pensar en cada posible situación que se puede presentar para así plasmar un curso de acción en el contrato. Las disputas futuras derivadas de un mal proceso de contratación pueden poner en riesgo la cadena de abastecimiento y con ello la misma existencia de la empresa.

\section{BIBLIOGRAFÍA}

CdS (2012). La Fórmula 1 apuesta por DHL. [En línea] Cadena de Suministro, Madrid, Sin info, 11 de mayo de 2012. 1p. [26 de 04 de 2013].

Klein, Naomi. (2002). No logo. El poder de las marcas. Madrid, Ediciones paidós Ibérica, S.A, 560 p. 
Langley, John (2013). 2013 Third Party Logistics Study: The State of Logistics Outsourcing. [En líneal Third Party Logistics Study: Annual Study on the State of Logistcs Outsourcing. Pennsylvania, Estados Unidos de America, Lisa Terry, 2013, 40 pp [22 de marzo de 2013].

Ministerio de Comercio Industria y Turismo. (2012).Gobierno da a conocerplan de acción para convertir a Colombia en plataforma exportadora de servicios [En línea] Boletín No. 49. Bogotá D.C., Mincomercio, 7 de diciembre de 2012, 21p [22 de 03 de 2013]

Pastrana, Andrés y otros (1999) Nueva legislación aduanera y su reglamentación. Decreto 2685. Título VIII: Régimen de transito aduanero, transporte multimodal, cabotaje y transbordo. Capítulo II: Transporte multimodal. Bogotá, D.C., Union LTDA, p 149-155.

Raja, K. (2000). Logistics and transportation: desing and planning. Berlin, Kluwer Academic Publishers, $240 \mathrm{p}$.

Rey, María. (2008). Encuesta Nacional de Logística-Colombia 2008. Resultados del benchmarketing Logístico. [En línea] Latin American Logistics Center. Atlanta - Estados Unidos, sin info de editor, 15 de diciembre de 2015, 130 p.[13 de mayo de 2013].

Robertson, Roland. (1995) Glocalización. [En línea] Global Modernities, Londres, Mike Featherstone y otros, 1995, p 25-45 [21 de septiembre de 2015]

Ruiz Olmedos, Sergio. (2013). Intermodal: panorama de un transporte en su última milla. En: énfasis logística, Vol. XIII, No. 149, mayo 2013, p. 28-33.

Saldarriaga Restrepo, Diego Luis (2009). Medición de desempeño en logística: indicadores en la gestion logistica. En: Zona Logistica, Vol. 09, No. 46, enero 2009 , p 22-27.

Semana. (2013). Cuestion de metodo. En: Así se Mueve Colombia: Un examen al transporte terrestre, aéreo, marítimo, férreo y fluvial del país, Vol. Sin info, No. Sin info, junio de 2013, 157p.

Tompkins Associates. (2010). Third Party Logistics. [En línea] Tompkins Associates Firm, Raleigh-Estados Unidos, 14 mayo 2010, p 52. [Marzo 2013].

Vargas M., Luis Daniel. (2013). Escoger un operador logístico: ¿Qué se debe hacer? [En línea] Revista de Logística, Bogotá, sin info de editor, sin info de fecha de publicación, lp. [Marzo 2013]. 